\title{
NUCLEAR-CYTOPLASMIC MALE STERILITY: SINGLE-POINT EQUILIBRIA VERSUS LIMIT CYCLES
}

\author{
P.-H. Gouyon, F. Vichot, and J. M. M. Van Damme* \\ Evolution et Systématique des Végétaux, Bâtiment 362, Université de Paris-sud Orsay, 91405 Orsay \\ Cedex, France; * Department of Botanical Ecology, Institute for Ecological Research, Boterhoekse \\ straat 22, P.O. Box 40, 6666 ZG Heteren, Holland
}

Submitted April 23, 1987; Revised February 23, 1988, and January 10, 1989; Accepted June 12, 1989

\begin{abstract}
The evolutionary dynamics of nuclear-cytoplasmic male sterility in a gynodioecious, self-incompatible species was examined through computer simulations of a deterministic model. In the model, two cytoplasmic types were assumed, each interacting with a specific, nuclear locus with one restorer and one sterility allele. A plant was male sterile when it was homozygous for the sterility allele at the locus corresponding to the cytoplasmic type it carried. Two additional fitness effects, which are the minimum required for maintenance of the nuclearcytoplasmic polymorphism, were considered: (1) the female fitness component of females relative to that of hermaphrodites, $x$, and (2) the cost of restoration, that is, the fitness of plants carrying a restorer gene together with the cytoplasmic type that is not restored by that allele. The parameter related to the cost of restoration, $a$, was assumed to act on male fertility. Two different types of stable nuclear-cytoplasmic polymorphism were obtained: the well-known single-point equilibria and limit cycles. We show how the occurrence of limit cycles as well as their shape depend on values of $x$ and $a$. Limit cycles are not a peculiarity of the model used; they also occur when some of the assumptions are relaxed. Models were tested with $a$ acting on female fertility and with recessiveness of the $x$ and $a$ effects of the restorer alleles. All models used were deterministic, but it can be inferred from the mechanism underlying limit cycles that finite populations can, in principle, also show cyclelike behavior, provided that some seed migration between populations occurs. The limit-cycle phenomenon may explain the negative correlation between the two cytoplasmic male-sterility types found in Plantago lanceolata and, more generally, any interpopulation variation in male-sterile frequencies, without invoking any ecological cause.
\end{abstract}

In the plant kingdom, individuals generally transmit their genes to the next generation through both pollen and ovules. The extent to which individuals function as males and females varies among and within species, resulting in a variety of sexual systems (Yampolsky and Yampolsky 1922; Carlquist 1966; Connor 1979). The ecological and evolutionary significance of this variation and the selective forces involved have been studied by many authors (e.g., Charlesworth and Charlesworth 1979; Bawa 1980; Ross 1980; Charnov 1982; Lloyd 1982; Queller 1983; Willson 1983; Sutherland and Delph 1984; Benjamin and Hainsworth 1986). In most studies, selection is considered to act on nuclear gene frequencies. An interesting form of sexual dimorphism in this respect is gynodioecy, the occurrence of male-sterile (i.e., female) and male-fertile (hermaphroditic) individuals in a population (Darwin 1877). In several well-studied species, the genetics of 
male sterility have been shown to involve both nuclear and cytoplasmic genes (Kheyr-Pour 1980; Van Damme 1983), and there is circumstantial evidence that this holds for many gynodioecious species (Charlesworth 1981). This complex mode of inheritance has important consequences for the evolutionary dynamics of the sexual system.

The primary action of cytoplasmic genes (in this article cytoplasmic genes are those transmitted only through female gametes) is mostly restricted to the construction and, therefore, to the functioning of the organelles that contain them, such as mitochondria and chloroplasts. In these processes, nuclear genes are often also involved (Grun 1976). Biologists have been accustomed to regarding cytoplasmic genes as "cooperating" with nuclear genes to ensure the correct functioning of the cell. This is a correct interpretation for many of the characters that have nuclear-cytoplasmic inheritance. It is also true in a general sense, because natural selection has retained genes in both the nucleus and the cytoplasm. However, Cosmides and Tooby (1981) have offered a more interesting evolutionary way of interpreting nuclear-cytoplasmic interactions between those genes that determine the production of male gametes (for a review of ideas, see also Frank 1989). Nuclear genes have been selected for determining a roughly equivalent investment into male and female functions, but this is not true for cytoplasmic genes. As long as male gametes lacking cytoplasmic genes have existed, selection in favor of cytoplasmic genes giving a higher production of such gametes could not have occurred, because the resources that are diverted from ovule production toward male function constitute a complete loss from the point of view of cytoplasmic gene transmission. Consequently, selection should favor cytoplasmic genes that are able to avoid this waste in favor of female function. From that point of view, the frequent occurrence of nuclear-cytoplasmic determination of male sterility in natural gynodioecious populations should not simply be viewed as the result of a "missed cooperation"' between nuclear and cytoplasmic genes, but as the expression of a fundamental evolutionary nuclear-cytoplasmic conflict (Gouyon and Couvet 1985; Frank 1989).

This conflict can be described as follows. A cytoplasmic gene blocking male function will be selected for, provided that female function is favored simultaneously, even if only slightly. In this context, any nuclear gene capable of restoring this cytoplasmic male sterility will be selected for because it allows the production of male gametes in the presence of a sterile cytoplasm. Such "resistance" genes have been found in every well-studied case of cytoplasmic male sterility (for agricultural species, see Edwardson 1970; for wild species, see Kheyr-Pour 1980, Van Damme 1983) and are called restorer genes. In fact, they have usually been found before the demonstration of cytoplasmic polymorphism, so that in early studies of gynodioecy the inheritance of male sterility has often been described as nuclear (Gouyon and Couvet 1985). There is a parallel between this system and the one found in plant-pathogen interactions. The cytoplasmic genome then corresponds to a pathogen with specific virulence genes, and the restorer gene to a resistance gene of the host.

An interesting example of nuclear-cytoplasmic gynodioecy is found in the perennial ribwort plantain, Plantago lanceolata $\mathrm{L}$. This species has two cytoplasmic 
types: each is either unrestored (i.e., determining female gender) or restored (i.e., determining hermaphrodite gender) by specific alleles of nuclear genes (Van Damme and Van Delden 1982; Van Damme 1983). In nuclear genes of this type, one allele is called a restorer allele and the other a sterility allele. In the present article, we attempt to model such a system. Previous theoretical studies of the maintenance of nuclear-cytoplasmic male sterility have shown that, for a joint polymorphism to be maintained, selection must act differently on the various genotypes determining a single phenotype. This effect has been suggested to originate in two ways. On the one hand, Delannay et al. (1981) and Charlesworth (1981) assumed the restorer gene had a detrimental effect when not combined with the cytoplasm it restores (comparable to the disadvantage of resistance genes in the absence of pesticides [Pasteur and Sinegre 1975] or pathogens [Olivieri 1985]). On the other hand, Ross and Gregorius (1985) assumed the existence of a direct overdominance effect at the restorer locus. Experimental evidence does not allow for discrimination between these two hypotheses. Partially male sterile forms in $P$. lanceolata are probably determined by heterozygous genotypes (Van Damme 1983) and may have a reproductive superiority over male steriles and hermaphrodites (Van Damme 1984), which would imply overdominance. However, precise genetic studies of a population containing almost only one cytoplasm showed that the proportion of restorers of the other cytoplasm was remarkably low (Van Damme 1986). This would be expected logically if restorers were counterselected when not in combination with their specific cytoplasm.

The models already published usually suppose that one of the cytoplasms (called normal) always gives fertile plants and the other gives either sterile or fertile plants according to the absence or presence of restorer genes. Ross and Gregorius (1985) have studied a case with two cytoplasmic types with a common restorer locus, in which each could give male sterility. Here we describe the properties of a model in which each of two cytoplasms can result in sterile or fertile plants according to its own nuclear restorer locus.

\section{THE MODEL}

The model is designed to resemble the situation in Plantago lanceolata. The species is self-incompatible (Ross 1973), and two morphologically distinct male sterility types, $\mathrm{MS}_{1}$ and $\mathrm{MS}_{2}$, can be distinguished (Van Damme and Van Delden 1982). Each male sterility type is expressed in plants with a specific cytoplasmic type and with sterility alleles at a number of restorer loci. Restorer loci are specific for each sterility type, although there are weak indications that there may be a locus in common for the two sterility types (Van Damme 1983). The number of restorer loci for $\mathrm{MS}_{1}$ and $\mathrm{MS}_{2}$ are at least five and three, respectively. In the model, however, only one restorer locus for each male sterility type will be considered.

The two cytoplasmic types, $C_{1}$ and $C_{2}$, are restored by nuclear loci 1 and 2 . The loci 1 and 2 are unlinked and bear alleles $R_{1} / r_{1}$ and $R_{2} / r_{2}$, with the dominant one $(R)$ being the restorer and the recessive allele $(r)$ being the sterility (or maintainer) allele. The sex of an individual is thus determined as shown in table 1 . 
TABLE 1

The Relationship between Sexual Phenotype AND GENOTYPE

\begin{tabular}{lcc}
\hline & $R_{2}-^{*}$ & $r_{2} r_{2}$ \\
\hline$R_{1}-$ & $\mathrm{MS}_{1 / H}$ & $\mathrm{MS}_{1 / \mathrm{MS}_{2}}$ \\
$r_{1} r_{1}$ & $H_{/ H}$ & $H_{/ \mathrm{MS}_{2}}$ \\
\hline
\end{tabular}

Note.-Rows and columns correspond to genotypes at loci 1 and 2, respectively. Upper left and lower right corners of each cell correspond to cytoplasms 1 and 2, respectively. $H$, Hermaphrodite; $\mathrm{MS}_{1}$ and $\mathrm{MS}_{2}$, respectively, refer to females of cytoplasm 1 and 2 .

* The second allele may be either $R_{2}$ or $r_{2}$.

For each of the 18 genotypes, a particular male and female fertility can be assumed. The only fixed parameters are the male fertilities of the females, which should be zero. As a consequence, 30 parameters are undetermined in this (simplified) model. It is assumed that mating is at random and that generations are nonoverlapping. The general recurrence formulas are given in the Appendix. A mathematical analysis of this system would be of interest but lies beyond the scope of this article. We take a biological point of view and wish only to point out the special qualitative properties of this system as shown by iterations of the recursive formulas. For this purpose, we confine ourselves to models with only two parameters varying. Furthermore, because even then a multitude of possibilities exist, we present one particular genetic model in detail, called here the standard model. We cite results of other models only when necessary to distinguish between peculiarities of the standard model and general phenomena.

Two parameters are considered.

1. The female fitness component of females relative to hermaphrodites $(\mathrm{x}) .-\mathrm{A}$ superiority in the female fitness component of females relative to that of hermaphrodites is generally found in gynodioecious species (Lloyd 1976; Van Damme 1984) and was explained by Darwin (1877) as a trade-off in resource allocation (he called this the compensation law). This supericrity may manifest itself in seed production per season (e.g., in Thymus vulgaris L.; Assouad et al. 1978; Couvet et al. 1985; Gouyon and Couvet 1985) or in adult survival (e.g., male sterility type $\mathrm{MS}_{1}$ in $P$. lanceolata; Van Damme and Van Delden 1984). Although female superiority in seed production per season and in adult survival rate have quantitatively different effects on female frequency (Van Damme and Van Damme 1986), their effects do not differ in a qualitative sense. In the standard model, it is assumed that there are no genotypic differences within sex phenotypes with respect to the female fitness component. This implies symmetry between cytoplasmic types. The parameter $x$ is chosen to represent the number of successful ovules (seeds) produced by a female divided by the corresponding value for hermaphrodites. In theory, $x$ can take any positive value. However, biological data on gynodioecious species (see above) suggest that $x$ is usually greater than one, that is, that females produce more seeds than do hermaphrodites. 
TABLE 2

\begin{tabular}{|c|c|c|}
\hline & $R_{2}-^{*}$ & $r_{2} r_{2}$ \\
\hline \multicolumn{3}{|l|}{ Male: } \\
\hline$R_{1}-$ & $1 / 1$ & $a_{/ 0}$ \\
\hline & $0 / a$ & $0_{/ 0}$ \\
\hline \multicolumn{3}{|l|}{ Female: } \\
\hline$r_{1} r_{1}$ & $x / 1$ & $\begin{array}{l}1 / x \\
x / x\end{array}$ \\
\hline $\begin{array}{l}\text { Note. } \\
\text { each cell } \\
\text { tively. } \\
* \text { The }\end{array}$ & $\begin{array}{l}\text { and low } \\
\text { cytopla }\end{array}$ & $\begin{array}{l}\text { ers of } \\
\text { espec- }\end{array}$ \\
\hline
\end{tabular}

2. The cost of restoration.-Like Delannay et al. (1981), we assume that selection acts against plants that carry a restorer gene together with the "other" cytoplasm, that is, the one not sensitive to that particular restorer gene. This "cost" attached to the ability to restore cytoplasmic male sterility when there is nothing to restore is assumed to act on male function. This assumption seems reasonable because a restorer gene affects male function in plants carrying the cytoplasm sensitive to this restorer gene. In the standard model, both the restorer effect (in the sensitive cytoplasm) and the cost effect (in the nonsensitive cytoplasm) are assumed to be dominant. In addition, like for $x$, symmetry between cytoplasmic types is assumed for these effects as well.

For technical reasons, the cost effect is expressed by a value $a$ representing the male fertility of nuclear genotype $r_{1} r_{1} R_{2}$ - (in which " - " denotes a "wild card" allele) in cytoplasm $C_{2}$ and of $r_{2} r_{2} R_{1}$ - in cytoplasm $C_{1}$, relative to the male fertility of the hermaphrodites carrying either restorer allele, $R_{1}$ or $R_{2}$. Consequently, the cost is not represented by $a$ itself but by $(1-a) / a$. The parameter $a$ can take any positive value, but it only represents a cost of restoration when $a>1$, and thus $(1-a) / a<1$.

Table 2 shows the matrices of male and female fitness for cytoplasms 1 and 2 , respectively, in the case of the standard model. Assumptions that were varied in other models are (1) the symmetry between cytoplasmic types in the values of $x$ and $a$, (2) the expression of $a$ in female fertility instead of in male fertility, and (3) the dominance or recessiveness of $x$ and $a$.

Combining the values in table 2 with the general formulas in the Appendix allows the calculation of the evolution of the sex-type frequencies from generation to generation. The criterion for ending a simulation run was a change in all gene and sex-type frequencies of less than $10^{-4}$ when measured over 500 consecutive generations. The maximum number of generations for a run was 5,000. Some of the runs that had not reached constant gene frequencies after 5,000 generations were tested further, up to a multiple of this number. All figures in this article have been drawn using a starting situation with the genotype $C_{1} R_{1} R_{1} r_{2} r_{2}$ at a frequency of 100 times that of each of the other genotypes; frequencies of the other genotypes equal one another. Such starting frequencies simulate the migra- 
tion of plants with a cytoplasmic type into a population where its restorer gene is (almost) absent. This resembles the situation observed for P. lanceolata (Van Damme 1986).

\section{RESULTS}

We start with the results of the standard model. All simulations resulted in one of the following situations:

1. Fixation of the restorer alleles at the two nuclear loci with or without fixation of one of the cytoplasmic types, resulting in a population of only hermaphroditic plants;

2. Fixation of one of the cytoplasmic types but maintenance of the polymorphism at the restorer locus of the remaining cytoplasmic type, resulting in a gynodioecious population with nuclear inheritance of male sterility;

3. Maintenance of joint polymorphism with all nuclear and cytoplasmic genes present, resulting in a gynodioecious population.

Result 3 confirms the hypothesis proposed by Delannay et al. (1981) and Charlesworth (1981) that a disadvantage of carrying a restorer together with the "other" cytoplasm is necessary to maintain a joint polymorphism in this type of model. Two different types of equilibrium situation appeared possible: (1) a single-point equilibrium in which all gene and sex-type frequencies converge to constant values, mostly within several hundreds of generations, or (2) limit cycles. Many runs gave a surprising result: shortly after the start of these runs the proportions of females as well as those of nuclear and cytoplasmic genes began fluctuating from generation to generation in undamped oscillations. The population became caught in a trajectory that reached a state of equilibrium (a limit cycle) in which the population oscillates forever.

Examples of these different outcomes of the simulations are presented in figure 1. Here the frequency of all male steriles, that is, $\mathrm{MS}_{1}+\mathrm{MS}_{2}$, is plotted against the frequency of cytoplasm $C_{1}$. In the case of a limit cycle, the result is a butterflylike figure (as shown in fig. 1a), in which the female-fitness parameter used is 1.7 and $a=2.0$. Lowering the value of $a$ first yields a cycle of a different shape $(a=1.6$; fig. $1 b)$, then a single-point equilibrium $(a=1.4$; fig. $1 c)$, and finally loss of polymorphism at both nuclear loci $(a=1.2$; fig. $1 d)$. Because of the imposed symmetry between cytoplasmic types, the equilibrium points in the last two cases should be at a cytoplasm $C_{1}$ frequency of $50 \%$. In figure $1 c$ equilibrium has clearly not yet been reached after 5,000 generations, but the variation in total MS frequency has been much reduced (to the order of $10^{-5}$, with a frequency of $2.4 \%$ ). In figure $1 d$, equilibrium is also attained slowly because of the deterministic nature of the model (cf. Charlesworth 1981). In a finite population, the loss of nuclear polymorphism would occur sooner and the remaining cytoplasmic polymorphism would disappear in the long run since it is "neutral.",

The sequence of figure $1 a-d$ is taken from an examination of the parameter space by a total of 6,000 randomly chosen combinations of $x$ and $a$. One thousand parameter combinations were drawn from the ranges $0-5$. The borderlines of the areas in this space yielding the various outcomes of the simulations with respect 

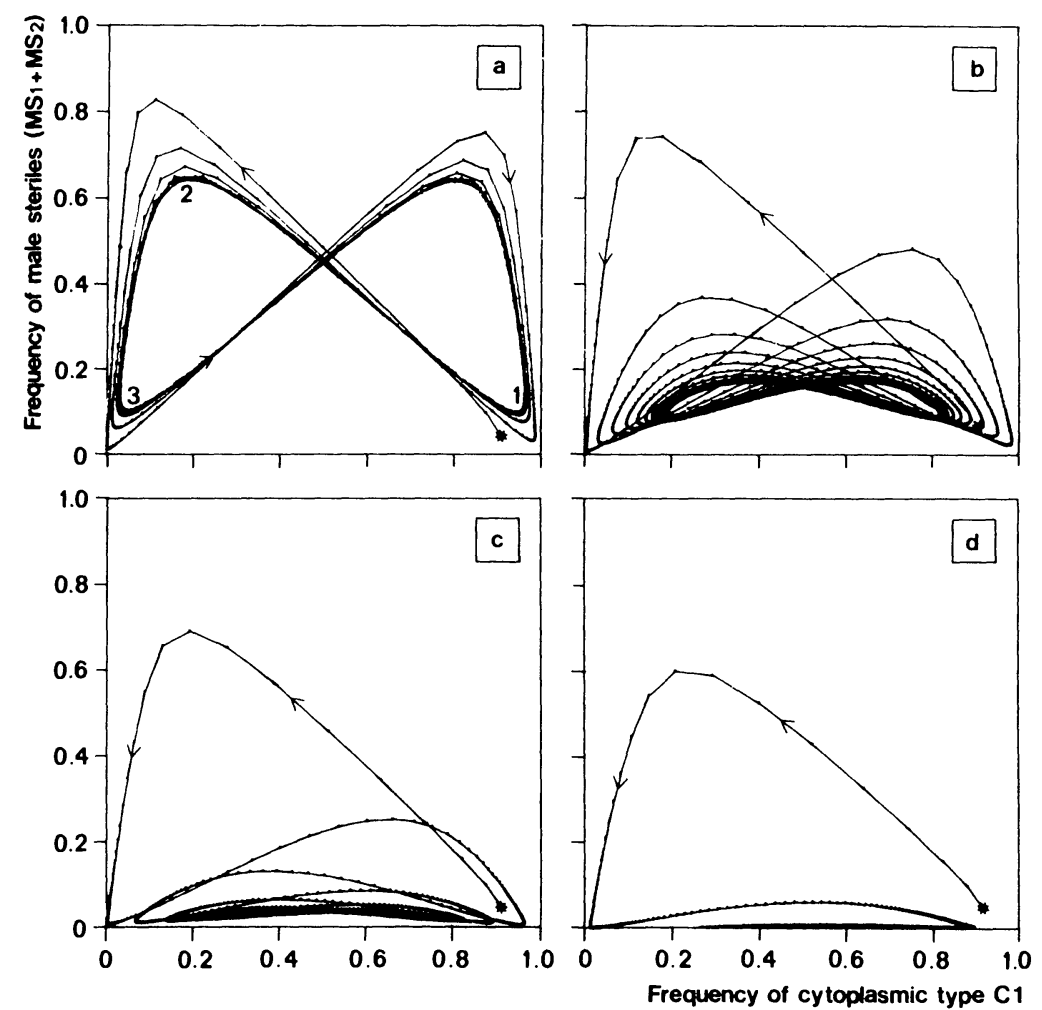

FIG. 1. - The frequency of all male steriles $\left(\mathrm{MS}_{1}+\mathrm{MS}_{2}\right)$ plotted against the frequency of cytoplasm $C_{1}$ during generations $0-5,000$ for $x=1.7$ combined with various $a$ values: $a, a$ $=2 ; b, a=1.6 ; c, a=1.4 ; d, a=1.2$ (these values of $x$ and $a$ are also used in fig. 5). These combinations of $x$ and $a$ are the two small circles in fig. 2. Each generation is shown except when the change in gene frequencies between consecutive generations is less than 0.002 .

to loss or maintenance of polymorphism were examined in more detail using another 5,000 runs. Furthermore, the stability of the different types of outcomes has been tested for various combinations of $x$ and $a$ in each area by starting from several origins and checking that the system ended in the same situation. Their description follows (fig. 2).

For $a \leq 1$ or $x \leq 1$, the joint three-locus, nuclear-cytoplasmic polymorphism cannot be maintained. This result is as expected because it simply shows that genes determining male sterility without giving compensatory advantage are lost. For the special case in which $a<1$ and $x \geq 2$, one of the cytoplasmic types is fixed, and the classic conditions of Lewis (1941) for the maintenance of nuclear polymorphism at the restorer locus of this cytoplasmic type are met.

When $x>1$ and $a>1$, the joint three-locus polymorphism seems to be maintained only when $x+a>3$. In this case, single-point equilibria are met for low values and limit cycles for high values of $x$ and $a$. The borderline between these 


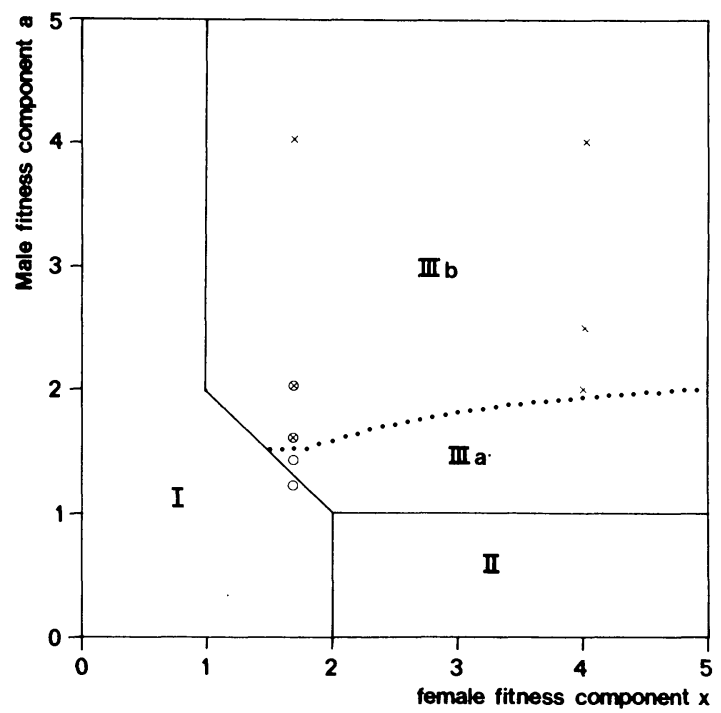

FIG. 2.-Summary of final states of simulation runs as a function of $x$ and $a$. The combinations of $x$ and $a$ give: I, hermaphroditism; II, nuclear gynodioecy; III, nuclear-cytoplasmic gynodioecy within (IIIa) single-point equilibria and (IIIb) limit cycles (for further explanation, see the text). Crosses, parameter combinations used in fig. 3; circles, parameter combinations used in fig. 1; two combinations have been used in both figs. 1 and 3 .

two situations (a Hopf bifurcation for difference equations; for an introduction to bifurcations, see Roughgarden 1979) is not a sharp one. In fact, when near the borderline, the cycles become vanishingly small (fig. 3), and it is impossible to decide whether the equilibrium corresponds to a single point or to an extremely small cycle. Analytical studies would be necessary to discriminate between these two situations. This is of no importance from a biological point of view because oscillations of the order of one in a thousand or less are negligible compared with random fluctuations in a natural population.

How the shape and size of a limit cycle depend on the value of $x$ and $a$ is illustrated in figure 3. Away from the borderline between cycles and point equilibria, the fluctuations in MS frequency are greater at lower $x$ (cf. fig. $3 a$ and $3 e$, fig. $3 b$ and $3 g$ ). Correspondingly, the period of the cycle, that is, the number of generations to complete one cycle, is larger at lower $x$. In figure 3 the period for the $x=1.7$ series (fig. $3 a-d$ ) is 52 or greater and the period for the $x=4$ series (fig. $3 e-h$ ) 26 or smaller. When the value of $a$ or $x$ is near the borderline (see also fig. 2), very small changes in the value of $a$ dramatically change the type of result obtained.

Biologically, the most interesting part of the parameter space is the square defined by $1 \leq x \leq 2$ and $1 \leq a \leq 2$. In the model presented here, limit cycles occur only for $a \geq 1.5$ (i.e., a cost of restoration of at least 0.33 ). Below that value, there is either hermaphroditism or stable gynodioecism, depending on $x$ (fig. 2). The size of the cycles in this square is small and partly beyond the 


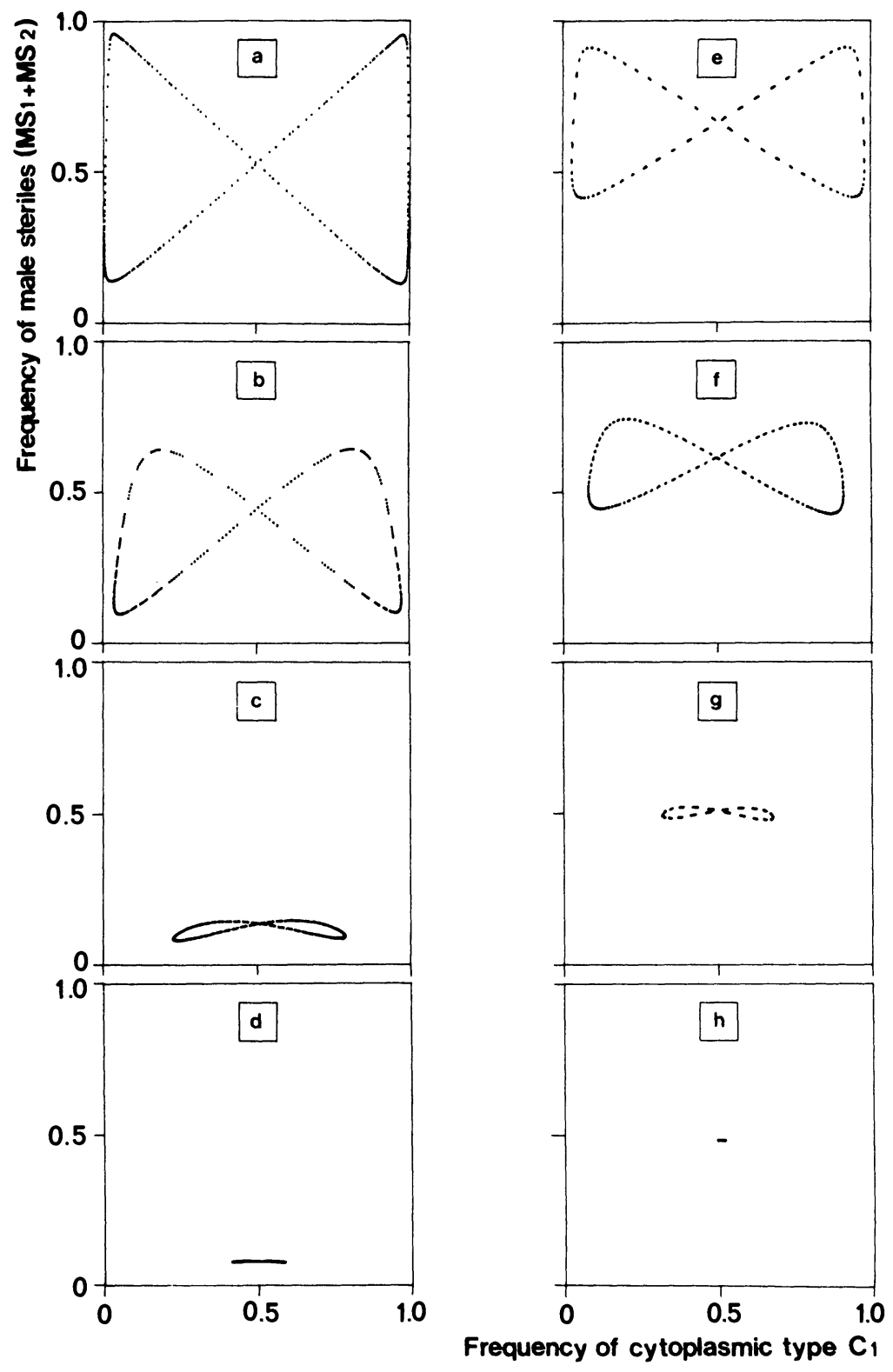

FIG. 3.-The shape of the limit cycle, expressed as the total male-sterile frequency plotted against the frequency of cytoplasm $C_{1}$, depending on $x$ and $a$ values. Fig. $3 a-d$ are drawn for $x=1.7$ and $a$ values of 4, 2, 1.6, and 1.55, respectively; fig. $3 e-h$ for $x=4$ and $a=4$, $2.5,2$, and 1.95, respectively. The positions of these combinations of $x$ and $a$ are indicated by small crosses in fig. 2 except for the values used in fig. $3 d$ and $3 h$, which are positioned almost on the borderline between limit cycles and single-point equilibria. 

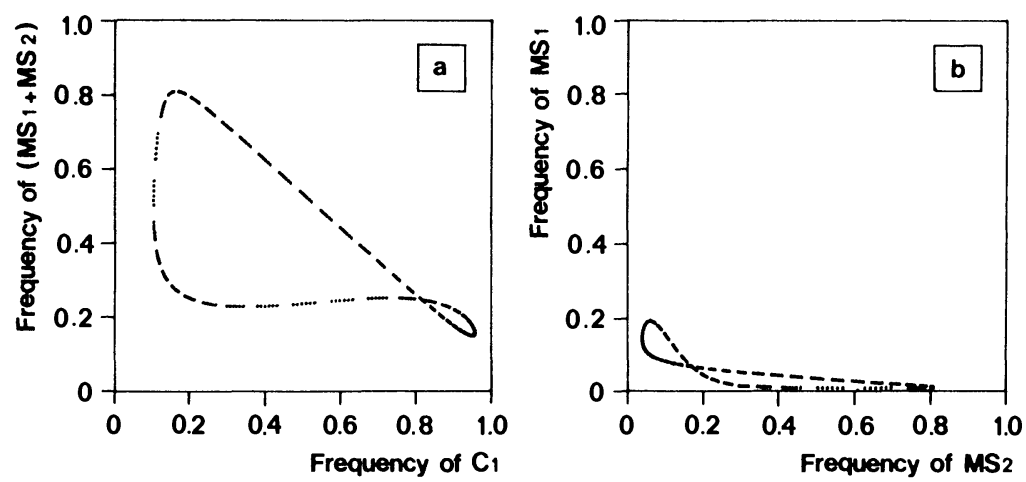

FIG. 4.-An asymmetrical limit cycle, expressed as the frequency of $\left(\mathrm{MS}_{1}+\mathrm{MS}_{2}\right)$ plotted against the frequency of cytoplasm $C_{1}$ (fig. $4 a$ ) and as the frequency of $\mathrm{MS}_{1}$ against that of $\mathrm{MS}_{2}$ (fig. 4b). Parameters $x=2$ and $a=2$; in addition, the female fertilities of genotypes with cytoplasm $C_{1}$ had been multiplied by 1.5 and their male fertilities by 0.8 .

detection level in field studies. In order to test whether the occurrence of limit cycles is a general feature of this type of model, and in particular in the biologically interesting part, additional models were run with some of the assumptions relaxed. First, models were tested without the symmetry in male or in female fertilities or in both. Limit cycles could easily be found, and their shape is asymmetric (see, e.g., fig. 4). Second, models were used with the cost of restoration imposed on female instead of male fertilities. If, in this case, the interaction of $x$ and $a$ in the $r_{1} r_{1} r_{2} r_{2}$ genotypes is assumed to be multiplicative, no limit cycles occurred at all. The other extreme was found in the third class of models in which the dominance relationships of $x$ and $a$ were varied: when compared to the standard model, the $x$ effect of allele $r_{1}$ in plants with cytoplasm $C_{1}$ is assumed to be dominant instead of recessive, and its $a$ effect in plants with cytoplasm $C_{2}$ is also dominant instead of recessive (see table 2). Virtually all combinations of $x$ and $a$ in the 1-2 range gave limit cycles, most of them with biologically relevant ranges in female frequencies. For example, a $16 \%$ range in female frequency over time occurs with $x=1.3$ and $a=1.2$, which implies a $30 \%$ female fitness advantage and a cost of restoration of $17 \%$. The results of most models were in between the extremes. In conclusion, therefore, the qualitative results of the standard model with respect to the existence of limit cycles also hold for various kinds of asymmetries between cytoplasmic types, for another way of expressing $a$, and for other dominance relationships of $x$ and $a$.

\section{DISCUSSION}

The chain of events in a limit cycle is illustrated in figure 5. At the start of the simulation run in the figure the predominant genotype is $C_{1} R_{1} R_{1} r_{2} r_{2}$. (In this section, we ignore heterozygous genotypes for simplicity's sake, but this is not essential.) Almost all of the few individuals carrying cytoplasm $C_{2}$ are male sterile $\left(C_{2} R_{1} R_{1} r_{2} r_{2}\right)$. Because male-sterile individuals have higher female fertility (table 


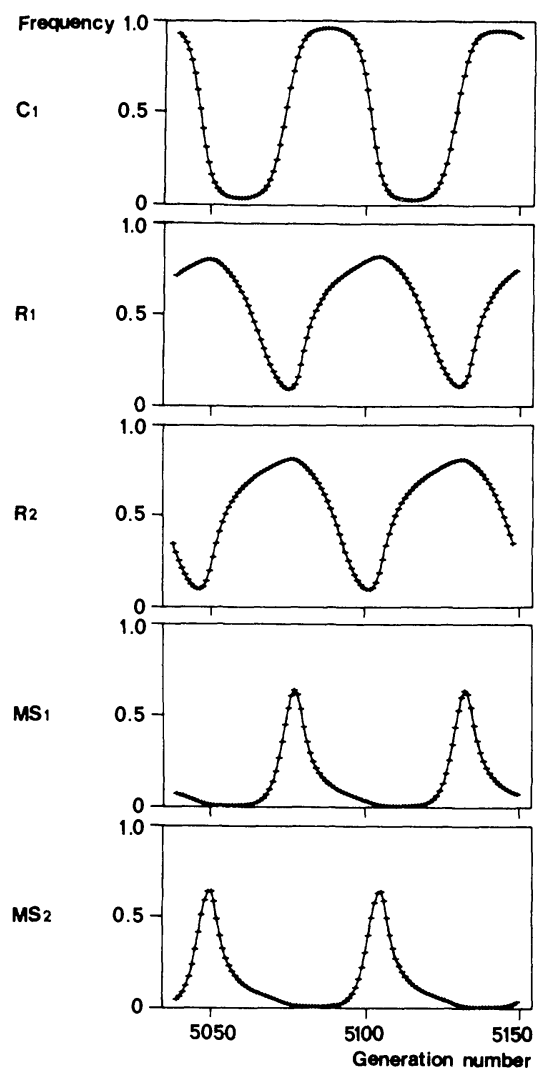

FIG. 5.-The frequencies of cytoplasmic $\left(C_{1}\right)$ and nuclear $\left(R_{1}\right.$ and $\left.R_{2}\right)$ gene frequencies and of the two male-sterility types $\left(\mathrm{MS}_{1}\right.$ and $\mathrm{MS}_{2}$ ) as a function of generation number in a simulation of the symmetric two-parameter model with the values $x=1.7$ and $a=2$. The equilibrium situation is shown from generation 5,040 onward for about 110 generations. Within the trajectory, each generation is plotted.

2), their frequency, and thus the frequency of cytoplasm $C_{2}$, increases sharply (fig. 5). The relation between these two frequencies is linear (fig. $1 a$, position 1 until position 2). After about 10 generations (fig. 5), the frequency of $C_{2}$ is so high that fitness relations among $C_{2}$ individuals become more important for frequency changes than those between $C_{1}$ individuals. In this second step, $C_{2} R_{1} R_{1} R_{2} R_{2}$ individuals are favored over $C_{2} R_{1} R_{1} r_{2} r_{2}$, resulting in a rapid decrease of the MS frequency and a rapid increase in the frequency of the restorer allele $R_{2}$ (fig. 5; fig. $1 a$, position 2 until position 3 ). In contrast to the previous step, this is essentially a within-cytoplasm $\left(C_{2}\right)$ process in which both male and female fertilities are important and fitnesses are frequency-dependent (cf. genotypes $C_{2} R_{1} R_{1}$ with $r_{2} r_{2}$ and $R_{2} R_{2}$ in table 2 ). When allele $R_{2}$ has become sufficiently common, individuals carrying the allele $r_{1}$ start increasing in frequency. This is caused by the higher male fertility of genotype $C_{2} r_{1} r_{1} R_{2} R_{2}$ as compared with that of $C_{2} R_{1} R_{1} R_{2} R_{2}$ (the 
cost of restoration; see table 2). During this third step, the population composition remains at position 3 in figure $1 a$ until the $r_{1}$ frequency approaches its maximum (see fig. 5). At that moment, the population composition is completely reversed when compared with the starting situation, and the process described above repeats itself with $C_{1}$ instead of $C_{2}$, and so on. The fact that the trajectories of $R_{1}$ and $R_{2}$ and of $\mathrm{MS}_{1}$ and $\mathrm{MS}_{2}$ have identical shapes and differ only by being out of phase is due to the symmetry in the parameter values of the particular model used here. In general the shapes differ from each other, but the trajectories are still out of phase because the cytoplasmic types alternate in frequency.

Whether limit cycles occur in natural gynodioecious populations is not known because of a lack of suitable observations. One could argue that, in particular, the low cytoplasm frequencies in the beginning of the development of a cycle prevent the establishment of a cycle in finite populations because of stochastic effects. However, even when the invasion of a cytoplasmic type results in its fixation, the population will show cyclelike behavior provided that some seed migration occurs. When the population in figure $1 a$ has arrived at position 3 , fixation of cytoplasm $C_{2}$ does not influence the changeover of the predominant nuclear genotype from $R_{1} R_{1} r_{2} r_{2}$ to $r_{1} r_{1} R_{2} R_{2}$. Migration of some individuals with cytoplasm $C_{1}$ is then sufficient to start the process again in the opposite direction until fixation of cytoplasm $C_{1}$ occurs, and so on.

Another argument with respect to the likelihood that cycles occur in nature concerns the values of $x$ and $a$ required. In the standard model, this depended mainly on the value of $a$, the relative male fertility of plants carrying a sterility allele together with the cytoplasm in which it is not expressed. Here limit cycles required $a$ to be higher than 1.5, which represents a cost of restoration of $33 \%$. Near this value, the cycles were small. In models with dominance instead of recessiveness of the $x$ and $a$ effects of one of the sterility alleles, limit cycles of detectable size were found for a female fitness advantage of $30 \%$ and a cost of restoration of $17 \%$. This female fitness advantage is well within the range of values reported in the literature, where differences between $50 \%$ and $100 \%$ are common (see Lloyd 1976; Van Damme 1984). In contrast, nothing is known of male-fertility variation in relation to a cost of restoration. There is, however, no a priori reason to believe that pollen fertility differences in the range of $15 \%-50 \%$ between restorer and nonrestorer alleles would be unrealistic, when differences larger than this size in female fertility have been found relatively often. Some indirect support comes from two monoecious species in which considerable male-fertility variation among clones has been found by Schoen and Stewart (1986) and Schoen and Cheliak (1987). In hermaphroditic Plantago lanceolata, a $25 \%$ difference in pollen production between two cytoplasmic types has been observed (Van Damme 1984). The occurrence of partial male steriles (Van Damme and Van Delden 1982) also contributes considerably to genotypic differences in male fertility.

Two other aspects of the parameters $a$ and $x$ considered in this study are the way in which $a$ is expressed, through male or through female fertility, and the dominance or recessiveness of both $a$ and $x$ relative to the nuclear male-sterility genes. Both aspects appeared relevant for the occurrence of limit cycles, but 
data are limited. Clearly, for insight into the relevance of limit cycles in natural populations and into the contribution of the forces represented by $x$ and $a$ to the dynamics of the system, detailed studies on the demography of gynodioecious populations, the fitness components involved, and the genetics of these components are required.

Cyclic behavior of populations has already been found in models of nuclearcytoplasmic male sterility with a single restorer locus (H. R. Gregorius and M. D. Ross, unpublished manuscript; J. M. M. Van Damme, unpublished data), but in these simpler models it seems to occur only in a very limited part of the parameter space. The present study shows that, when a specific restorer locus corresponds to each cytoplasmic type, the occurrence of these oscillations becomes the rule rather than the exception. An intriguing parallel exists between these cycles and those found in studies of host-parasite systems (Flor 1955). Any new gene in the pathogen (cytoplasm) enhancing its ability to attack the host induces selection for a new resistance (restorer) gene; once this gene has been established, selection favors another pathogenic form, and so on. From this point of view, the limit cycles presented here represent the ultimate dynamic expression of the intragenomic conflict described by Cosmides and Tooby (1981).

An important consequence of the possible existence of limit cycles is that variation in the frequencies of male steriles in gynodioecious species does not necessarily correspond to any adaptive or ecological cause. Two populations can be exactly equivalent with respect to fitness relationships between sex types and exhibit very different frequencies of male steriles just because they are in different parts of the same cycle. In that case, there is no point in trying to explain high frequencies of male steriles by an equilibrium situation resulting from the action of ecological factors (Gouyon et al. 1983). Erratic female frequencies, like those reported by Baker (1963), who found a $P$. lanceolata population in which $70 \%$ of the members were male steriles, could be explained by our model, because in the model periods with peak frequencies of male steriles are typically short, with steep frequency changes alternating with long periods of little change at the phenotypic level and lower male-sterile frequencies. Krohne et al. (1980) found a larger decrease in the male-sterile frequency from one year to the next in populations of $P$. lanceolata when the initial frequency was higher, as would be expected from the model. Limit cycles could also provide an explanation for the negative correlation between $\mathrm{MS}_{1}$ and $\mathrm{MS}_{2}$ found in the same species, because the two male-sterility types each represent a different cytoplasmic type (cf. Van Damme and Van Delden 1982, fig. 2, with fig. $4 b$ here). Finally, the decline in male-sterile frequency in Thymus vulgaris with an increase in population age (Dommée and Jacquard 1985) can be interpreted according to this model. In conclusion, the nuclear-cytoplasmic conflict leads to systems that have their own dynamics and that can modify (even endlessly) the structure of the population without any change in the environment. 


\section{APPENDIX}

General Description of the Computer Model

The system contains two cytoplasmic types $\left(C_{1}\right.$ and $\left.C_{2}\right)$ and two specific restorer loci (with alleles $R_{1} / r_{1}$ and $R_{2} / r_{2}$, respectively). The frequencies and fertilities of the different nuclear genotypes are denoted by two indices: the first $(i)$ refers to the genotype at the locus $R_{1} / r_{1}$ and the second $(j)$ to that at the locus $R_{2} / r_{2}$. In sporophytes (diploid individuals), the indices take the values 1,2 , and 3 for genotypes $R R, R r$, and $r r$, respectively. In gametes (haploid), the values of the indices are 1 and 2 for genotypes $R$ and $r$, respectively.

The frequency of individuals with nuclear genotype $(i, j)$ and with cytoplasmic type $C_{1}$ are designated $a_{i j} ; b_{i j}$ is the corresponding term for plants with cytoplasmic type $C_{2}$. The frequency of ovules with haploid genotype $(i, j)$ and with cytoplasmic type $C_{1}$ are called $r_{i j}$, the corresponding frequency of ovules with cytoplasmic type $C_{2} s_{i j}$, and the frequencies of pollen $p_{i j}$ (without any distinction of cytoplasmic type because pollen grains are assumed not to transmit any cytoplasm). The female fertilities are denoted by $f_{i j}$ and $g_{i j}$ in plants with cytoplasmic type $C_{1}$ and $C_{2}$, respectively, and the male fertilities by $m_{i j}$ and $n_{i j}$, respectively. The evolution of gene frequencies can be computed by the following set of formulas.

First, the gametic frequencies are calculated by adding the contributions of each genotype, using its frequency, its female and male fertilities, and Mendelian segregation ratios.

Ovules with cytoplasmic type $C_{1}$ :

$$
\begin{aligned}
& k_{1} r_{11}=f_{11} a_{11}+1 / 2 f_{12} a_{12}+1 / 2 f_{21} a_{21}+1 / 4 f_{22} a_{22} \\
& k_{1} r_{12}=f_{13} a_{13}+1 / 2 f_{12} a_{12}+1 / 2 f_{23} a_{23}+1 / 4 f_{22} a_{22} \\
& k_{1} r_{21}=f_{31} a_{31}+1 / 2 f_{21} a_{21}+1 / 2 f_{32} a_{32}+1 / 4 f_{22} a_{22} \\
& k_{1} r_{22}=f_{33} a_{33}+1 / 2 f_{23} a_{23}+1 / 2 f_{32} a_{32}+1 / 4 f_{22} a_{22}
\end{aligned}
$$

Ovules with cytoplasmic type $C_{2}$ :

$$
\begin{aligned}
& k_{1} s_{11}=g_{11} b_{11}+1 / 2 g_{12} b_{12}+1 / 2 g_{21} b_{21}+1 / 4 g_{22} b_{22} \\
& k_{1} s_{12}=g_{13} b_{13}+1 / 2 g_{12} b_{12}+1 / 2 g_{23} b_{23}+1 / 4 g_{22} b_{22} \\
& k_{1} s_{21}=g_{31} b_{31}+1 / 2 g_{21} b_{21}+1 / 2 g_{32} b_{32}+1 / 4 g_{22} b_{22} \\
& k_{1} s_{22}=g_{33} b_{33}+1 / 2 g_{23} b_{23}+1 / 2 g_{32} b_{32}+1 / 4 g_{22} b_{22}
\end{aligned}
$$

Pollen:

$$
\begin{aligned}
k_{2} p_{11}= & m_{11} a_{11}+1 / 2 m_{12} a_{12}+1 / 2 m_{21} a_{21}+1 / 4 m_{22} a_{22} \\
& +n_{11} b_{11}+1 / 2 n_{12} b_{12}+1 / 2 n_{21} b_{21}+1 / 4 n_{22} b_{22} \\
k_{2} p_{12}= & m_{13} a_{13}+1 / 2 m_{12} a_{12}+1 / 2 m_{23} a_{23}+1 / 4 m_{22} a_{22} \\
& +n_{13} b_{13}+1 / 2 n_{12} b_{12}+1 / 2 n_{23} b_{23}+1 / 4 n_{22} b_{22} \\
k_{2} p_{21}= & m_{31} a_{31}+1 / 2 m_{21} a_{21}+1 / 2 m_{32} a_{32}+1 /{ }_{4} m_{22} a_{22} \\
& +n_{31} b_{31}+1 / 2 n_{21} b_{21}+1 / 2 n_{32} b_{32}+1 / 4 n_{22} b_{22} \\
k_{2} p_{22}= & m_{33} a_{33}+1 / 2 m_{23} a_{23}+1 / 2 m_{32} a_{32}+1 / 4 m_{22} a_{22} \\
& +n_{33} b_{33}+1 / 2 n_{23} b_{23}+1 / 2 n_{32} b_{32}+1 / 4 n_{22} b_{22},
\end{aligned}
$$

where $k_{1}$ and $k_{2}$ are normalizing factors such that $\Sigma\left(r_{i j}+s_{i j}\right)=1$ and $\Sigma p_{i j}=1$. 
Next-generation zygote frequencies (denoted by a prime) follow by adding the appropriate product terms of gametic frequencies:

$$
\begin{aligned}
& a_{11}^{\prime}=r_{11} p_{11} \\
& a_{12}^{\prime}=r_{11} p_{12}+r_{12} p_{11} \\
& a_{21}^{\prime}=r_{21} p_{11}+r_{11} p_{21} \\
& a_{22}^{\prime}=r_{11} p_{22}+r_{22} p_{11}+r_{21} p_{12}+r_{12} p_{21} \\
& a_{13}^{\prime}=r_{12} p_{12} \\
& a_{23}^{\prime}=r_{12} p_{22}+r_{22} p_{12} \\
& a_{31}^{\prime}=r_{21} p_{21} \\
& a_{32}^{\prime}=r_{21} p_{22}+r_{22} p_{21} \\
& a_{33}^{\prime}=r_{22} p_{22} \\
& b_{11}^{\prime}=s_{11} p_{11} \\
& b_{12}^{\prime}=s_{11} p_{12}+s_{12} p_{11} \\
& b_{21}^{\prime}=s_{21} p_{11}+s_{11} p_{21} \\
& b_{22}^{\prime}=s_{11} p_{22}+s_{22} p_{11}+s_{21} p_{12}+s_{12} p_{21} \\
& b_{13}^{\prime}=s_{12} p_{12} \\
& b_{23}^{\prime}=s_{12} p_{22}+s_{22} p_{12} \\
& b_{31}^{\prime}=s_{21} p_{21} \\
& b_{32}^{\prime}=s_{21} p_{22}+s_{22} p_{21} \\
& b_{33}^{\prime}=s_{22} p_{22}
\end{aligned}
$$

The relation between the general notation above and the model used in the article can be obtained by comparing the notation with that in the tables. The parameter $x$, for example, is represented by $f_{3 j}$ and $g_{i 3}$, and $a$ by $m_{13}, m_{23}, n_{31}$, and $n_{32}$.

\section{LITERATURE CITED}

Assouad, M. W., B. Dommée, R. Lumaret, and G. Valdeyron. 1978. Reproductive capacities in the sexual forms of the gynodioecious species Thymus vulgaris L. Botanical Journal of the Linnean Society 77:29-39.

Baker, H. G. 1963. Evolutionary mechanisms in pollination biology. Science (Washington, D.C.) 139:877-883.

Bawa, K. S. 1980. Evolution of dioecy in flowering plants. Annual Review of Ecology and Systematics 11:15-39.

Benjamin, R. B., and F. R. Hainsworth. 1986. Sex change with inbreeding: experiments on separate versus combined sexes. Evolution 40:843-855.

Carlquist, S. 1966. The biota of long distance dispersal. IV. Genetic systems in the floras of oceanic islands. Evolution 20:433-455.

Charlesworth, D. 1981. A further study of the problem of the maintenance of females in gynodioecious species. Heredity 46:27-39.

Charlesworth, D., and B. Charlesworth. 1979. The evolutionary genetics of sexual systems in flowering plants. Proceedings of the Royal Society of London B, Biological Sciences 205:513-530.

Charnov, E. L. 1982. The theory of sex allocation. Princeton University Press, Princeton, N.J.

Connor, H. E. 1979. Breeding systems in grasses: a survey. New Zealand Journal of Botany 17:547-574. 
Cosmides, L. M., and J. Tooby. 1981. Cytoplasmic inheritance and intragenomic conflict. Journal of Theoretical Biology 89:83-129.

Couvet, D., P. H. Gouyon, F. Kjellberg, I. Olivieri, D. Pomente, and G. Valdeyron. 1985. De la metapopulation au voisinage: la génétique des populations en deséquilibre. Genetique, Selection, Evolution 17:407-414.

Darwin, C. 1877. Different forms of flowers on plants of the same species. J. Murray, London.

Delannay, X., P. H. Gouyon, and G. Valdeyron. 1981. Mathematical study of the evolution of gynodioecy with cytoplasmic inheritance under the effect of a nuclear restorer gene. Genetics 99:169-181.

Dommée, B., and P. Jacquard. 1985. Gynodioecy in thyme, Thymus vulgaris L.: evidence from successional populations. Pages 141-164 in P. Jacquard, G. Heim, and J. Antonovics, eds. Genetic differentiation and dispersal in plants. Springer, Berlin.

Edwardson, J. R. 1970. Cytoplasmic male sterility. Botanical Review 36:341-420.

Flor, H. H. 1955. Host-parasite interaction in flax rust: its genetics and other implications. Phytopathology 45:680-685.

Frank, S. A. 1989. The evolutionary dynamics of cytoplasmic male sterility. American Naturalist 133:345-376.

Gouyon, P. H., and D. Couvet. 1985. Selfish cytoplasm and adaptation: variations in the reproductive system of thyme. Pages 299-319 in J. Haeck and J. W. Woldendorp, eds. Structure and functioning of plant populations. II. North-Holland, Amsterdam.

Gouyon, P. H., R. Lumaret, G. Valdeyron, and P. Vernet. 1983. Reproductive strategies and disturbance by man. Pages 214-225 in H. Mooney, ed. Ecosystems and disturbance. Springer, Berlin.

Grun, P. 1976. Cytoplasmic genetics and evolution. Columbia University Press, New York.

Kheyr-Pour, A. 1980. Nucleo-cytoplasmic polymorphism for male sterility in Origanum vulgare L. Journal of Heredity 71:253-260.

Krohne, D. T., I. Baker, and H. G. Baker. 1980. The maintenance of the gynodioecious breeding system in Plantago lanceolata L. American Midland Naturalist 103:269-279.

Lewis, D. 1941. Male sterility in natural populations of hermaphrodite plants. New Phytologist 40:56-63.

Lloyd, D. G. 1976. The transmission of genes via pollen and ovules in gynodioecious angiosperms. Theoretical Population Biology 9:299-316.

1982. Selection of combined versus separate sexes in seed plants. American Naturalist 120:571-585.

Olivieri, I. 1985. Effect of Puccinia cardui pycnocephali on slender thistles. Weed Science 32:508-510.

Pasteur, N., and G. Sinegre. 1975. Esterase polymorphism and sensitivity to Dursban-organophorus insecticides in Culex pipens pipens populations. Biochemical Genetics 13:789-803.

Queller, D. C. 1983. Sexual selection in hermaphroditic plants. Nature (London) 305:765-766.

Ross, M. D. 1973. Inheritance of self-incompatibility in Plantago lanceolata. Heredity 30:169-176. 1980. The evolution and decay of overdominance during the evolution of gynodioecy, subdioecy and dioecy. American Naturalist 116:607-620.

Ross, M. D., and H. R. Gregorius. 1985. Selection with gene-cytoplasm interactions. II. Maintenance of gynodioecy. Genetics 109:427-439.

Roughgarden, J. 1979. Theory of population genetics and evolutionary ecology: an introduction. Macmillan, New York.

Schoen, D. J., and W. M. Cheliak. 1987. Genetics of the polycross. II. Male fertility variation in Norway spruce, Picea abies (L.) Karst. Theoretical and Applied Genetics 74:554-559.

Schoen, D. J., and S. C. Stewart. 1986. Variation in male reproductive investment and male reproductive success in white spruce. Evolution 40:1109-1120.

Sutherland, S., and L. F. Delph. 1984. On the importance of male fitness in plants: patterns of fruit set. Ecology 65:1093-1104.

Van Damme, J. M. M. 1983. Gynodioecy in Plantago lanceolata L. II. Inheritance of three malesterility types. Heredity 50:253-273.

1984. Gynodioecy in Plantago lanceolata L. III. Sexual reproduction and the maintenance of male steriles. Heredity 52:77-93. 
1986. Gynodioecy in Plantago lanceolata L. V. Frequencies and spatial distribution of nuclear and cytoplasmic genes. Heredity 56:355-364.

Van Damme, J. M. M., and R. Van Damme. 1986. On the maintenance of gynodioecy: Lewis' result extended. Journal of Theoretical Biology 121:339-350.

Van Damme, J. M. M., and W. Van Delden. 1982. Gynodioecy in Plantago lanceolata L. I. Polymorphism for plasmon type. Heredity 49:303-318.

1984. Gynodioecy in Plantago lanceolata L. IV. Fitness components of sex types in different life-cycle stages. Evolution 38:1326-1336.

Willson, M. F. 1983. Plant reproductive ecology. Wiley, New York.

Yampolsky, C. R., and H. Yampolsky. 1922. Distribution of the sex forms in the phanerogamic flora. Bibliotheca Genetica 3:1-62. 